\title{
ACTIVIDAD ANTIOXIDANTE, HIPOLIPEMIANTE $Y$ ANTIPLAQUETARIA DEL TOMATE (Solanum lycopersicum L.) Y EL EFECTO DE SU PROCESAMIENTO Y ALMACENAJE
}

\author{
ANTIOXIDANT, LIPID-LOWERING AND ANTIPLATELET \\ ACTIVITY OF TOMATO (Solanum lycopersicum L.) \\ AND THE EFFECT OF ITS PROCESSING AND STORAGE
}

\author{
Iván Palomo G. (1), Eduardo Fuentes Q. (1), Gilda Carrasco S. (2), \\ Daniel González R. (3), Rodrigo Moore-Carrasco (1)
}

(1) Programa de Investigación en Factores de Riesgo de Enfermedades Cardiovasculares (PIFRECV), Departamento de Bioquímica Clínica e Inmunohematología, Facultad de Ciencias de la Salud,

Universidad de Talca, Talca, Chile.

(2) Departamento de Horticultura, Facultad de Ciencias Agrarias,

Universidad de Talca. Talca, Chile.

(3) Departamento de Ciencias Básicas Biomédicas, Facultad de Ciencias de la Salud,

Universidad de Talca, Talca, Chile.

\begin{abstract}
Cardiovascular diseases (CVD) are the main cause of mortality worldwide. To prevent CVD it is recomended to quit smoking, the practice of physical activity and the consumption of healthy food. In this context, numerous studies have shown the importance of a frequent consumption of fruits and vegetables (at least 5 a day). It has been described an inverse relationship between vegetables consumption and the risk of developing CVD, which is mainly explained by its antioxidant activity, and in some cases lipid-lowering and antiplatelet effects. In this sense, the increase in regular consumption of tomato (Solanum lycopersicum L.) and related products, can improve the some cardiovascular parameters. The current lifestyle favors the consumption of processed foods, a situation that may affect the stability of tomato components and their physicochemical properties. This review addresses the antioxidant activities, lipid-lowering and antiaggregant properties of tomato, as well as the effect of processing and storage. Additionally, a summary of some patents associated with beneficial effects on health. As bibliographic source www.pubmed.org was mainly used, the terms used in the search were: antiplatelet, tomato, and platelet, antioxidant, among others, then search the full texts of items of common interest.
\end{abstract}

Keywords: Tomatoes, antioxidant, lipid lowering, antiplatelet, cardiovascular diseases, processing, patents.

Este trabajo fue recibido el 5 de Julio de 2010 y aceptado para ser publicado el 12 de Noviembre de 2010.

\section{INTRODUCCIÓN}

Las enfermedades no transmisibles (ENT), entre las que se encuentran las enfermedades cardiovasculares (ECV), representan un problema de salud pública a nivel mundial $(1,2)$.

Las ECV (infarto agudo de miocardio, enfermedad cerebro vascular y trombosis arterial periférica) desde el punto de vista patogénico, se inician por un proceso aterotrombótico (3), en el cual el endotelio (4), plaquetas (5) y monocitos (6) juegan un importante papel, generando una respuesta inflamatoria $(7,8)$. El endotelio disfuncional, favorecido por el aumento de las especies reactivas del oxígeno (ROS), genera un estado pro-aterotrombótico en el que las plaquetas tienen una importante participación (9-11).

Varios estudios han mostrado la alta prevalencia de factores de riesgo de ECV en la población adulta chilena (12-15). En el desarrollo de las ENT participan 
especialmente el tabaquismo, el sedentarismo y la alimentación no saludable (16). Respecto a esto último, numerosos estudios han demostrado que el consumo regular de frutas y hortalizas (al menos 5 porciones al día) previene el desarrollo de ECV (17-22).

Esta revisión se refiere a la actividad antioxidante, hipolipemiante y antiplaquetaria del tomate (Solanum lycopersicum $L$ ) y al efecto que su procesamiento y almacenamiento industrial tiene sobre dichas actividades. Finalmente se mencionan algunas innovaciones sobre extractos de tomates con actividad antioxidante, hipolipemiante y antiagregante plaquetaria.

\section{CARACTERÍSTICAS DE LA ESPECIE Y MEJORAMIENTO GENÉTICO}

El tomate es una planta dicotiledónea perteneciente a la familia de las solanáceas (23). Es una de las principales hortalizas cultivada en Chile, concentrándose el $88 \%$ de la superficie productiva entre la región V y VII (24).

El tomate es una rica fuente de potasio, ácido fólico, beta caroteno, vitamina $\mathrm{C}$, vitamina $\mathrm{E}$, flavonoides $\mathrm{y}$ licopeno $(25,26)$. Varias de estas moléculas presentan actividad antioxidante (27), la que puede variar según el cultivar (25).

El desarrollo de nuevas variedades de tomates ha permitido seleccionar algunas con propósitos agroindustrial, las que en relación a las de consumo en fresco, presentan mayor contenido de licopeno (28). Otras características, tales como rendimiento, resistencia a enfermedades y a estrés abiótico y calidad de frutos (atributos sensoriales: sabor, viscosidad y color; y calidad de fruto: materia seca, sólidos solubles y pH), se han mejorado genéticamente con el propósito de favorecer el tomate para proceso (29). Además, se busca la mejora genética de características agronómicas tales como el tamaño compacto y uniforme de las plantas para realizar una cosecha mecanizada $(30,31)$.

\section{ACTIVIDAD ANTIOXIDANTE, HIPOLIPEMIANTE Y ANTIAGREGANTE PLAQUETARIA DEL TOMATE}

\section{Actividad antioxidante}

El consumo regular de tomate y sus derivados puede ayudar a mejorar la capacidad de defensa del organismo frente al estrés oxidativo (32-37), debido a su contenido en flavonoides, carotenoides y polifenoles, (potentes antioxidantes) (38), contenido que puede variar según el cultivar (39).

Estudios realizados en humanos muestran que el consumo de tomate y productos a base de esta hortaliza, por un tiempo determinado, puede aumentar la protección del ADN ante el daño inducido por ROS $(27,40-42)$. Este efecto que puede explicarse por la sinergia de los diferentes antioxidantes presentes en el tomate (43). Adicionalmente, una dieta rica en productos procesados de tomate (salsa, pasta, sopa, jugo y conserva) permite obtener una disminución significativa de la peroxidación de lípidos y oxidación de $\operatorname{LDL}(40,44)$. En experimentos realizados en ratas se observó que el consumo de diferentes suplementos de tomates disminuyó la susceptibilidad a la oxidación de microsomas hepáticos (45), la peroxidación lipídica (46) y el estrés oxidativo (33).

Originalmente la actividad antioxidante del tomate se asoció principalmente a su contenido de licopeno. Sin embargo, el tomate es fuente de ácido ascórbico, polifenoles y flavonoides que también tienen esta actividad, y que probablemente contribuyen a los efectos observados.

En la tabla 1 se resume el diseño y resultados de varios estudios que analizaron la actividad antioxidante del tomate.

\section{Actividad hipolipemiante}

Estudios en humanos muestran que el consumo de derivados del tomate puede reducir el nivel de colesterol total (47 - 49), el nivel de LDL (50) y aumentar el nivel de HDL, (51), con menor efecto sobre los triglicéridos. Los mecanismos por los cuales componentes del tomate afectan el metabolismo, absorción o excreción del colesterol y las lipoproteínas se desconocen. Sin embargo, los derivados del tomate han mostrado su capacidad de inhibir la oxidación de la LDL, lo que probablemente se deba a su contenido de moléculas antioxidantes.

En la tabla 2 se resume el diseño y resultados de varios estudios en que se analizó la actividad hipolipemiante del tomate.

\section{Actividad antiagregante plaquetaria}

La actividad antiagregante del tomate puede contribuir a reducir eventos aterotrombóticos (52). En nuestro laboratorio se observó que la actividad antiagregante plaquetaria in vitro de extractos acuoso y metanólico de tomates híbridos tipo racimo, a una concentración de $1 \mathrm{mg} / \mathrm{mL}$, fue de $\sim 30 \%$ y $35 \%$, respectivamente, usando ADP como agonista agregante (53). Estos resultados son consistentes con las observaciones obtenidas tanto en ratones (52) como en humanos (54-57). El mecanismo de acción por el cual fracciones de tomate inhiben la agregación plaquetaria aun no ha sido dilucidado. Se ha sugerido que la adenosina (u otro nucleosido) podría ser responsable de esta inhibición, por un mecanismo al parecer independiente de la generación de cAMP y tromboxanos (57). 


\section{TABLA 1}

\section{Estudios que muestran actividad antioxidante del tomate.}

\section{Sujetos/animales}

19 sujetos sanos

\begin{abstract}
9 mujeres sanas (edad promedio de $25.2 \pm 2.2$ años, $\mathrm{IMC}=20.2 \pm 1.6$ $\mathrm{kg} / \mathrm{m} 2)$.

9 mujeres sanas (edad promedio de $25,4 \pm 2.2$ años, $\mathrm{IMC}=20,3 \pm 1,5$ $\mathrm{Kg} / \mathrm{m} 2$ ).
\end{abstract}

\section{3 grupos de 6 ratas} Sprague-Dawley macho (90-100 g).

12 mujeres sanas (IMC= 20,6 \pm 1,8 $\mathrm{kg} / \mathrm{m} 2)$.

26 sujetos sanos.

40 ratas

Wistar macho (180-200 g).

180 codornices (edad promedio de 10 días).
Tomate o derivados

Consumos de diferentes dietas (Salsa de tomate, jugo de tomate y oleorresina de tomate) al día por una semana.

Consumo de $25 \mathrm{~g}$ de puré de tomate ( $7 \mathrm{mg}$ de licopeno) al día por dos semanas.

Dieta de espinacas $(150 \mathrm{~g})+$ puré de tomate $(25 \mathrm{~g})$ al día durantes 3 semanas.

Cada grupo con diferentes dietas (extracto de tomate al 0\% (I), al $5 \%$ (II) y al $10 \%$ (III)) durante 9 semanas.

Una dieta estándar para 1 semana, seguido por el consumo de $100 \mathrm{~g}$ de tomate crudo, 60 $\mathrm{g}$ de salsa de tomate y $15 \mathrm{~g}$ de pasta de tomate al día durante 3 semanas.

$250 \mathrm{~mL}$ de bebida (6 $\mathrm{mg}$ de licopeno, $4 \mathrm{mg}$ fitoeno, $3 \mathrm{mg}$ fitoflueno, $1 \mathrm{mg}$ de $\beta$-caroteno y 1,8 $\mathrm{mg}$, de $\alpha$-tocoferol) o una bebida placebo, al día por 26 días.

Cada grupo con diferentes dietas (grupo RT, grupo YT, grupo LB y grupo LVED) durante 6 semanas.

Suplemento de tomate en polvo al $2,5 \%$ y $5 \%$.

Determinaciones

Resultados

Referencia

Oxidación LDL.

Disminución de la 44 peroxidación de lípidos y oxidación de LDL.

Resistencia de linfocitos al estrés oxidativo.

Resistencia de ADN al estrés oxidativo.

Efecto antioxidante.

Daño oxidativo de lípidos y ADN de linfocitos.

Daño oxidativo de ADN de linfocitos.

Estrés oxidativo.

Disminución del estrés oxidativo, ex vivo.

Protección del ADN contra el estrés oxidativo, ex vivo. y de la resistencia de los linfocitos a estrés oxidativo, ex vivo.

Protección del ADN contra el estrés oxidativo, ex vivo.

Disminución de la susceptibilidad a la oxidación de microsomas hepáticos, in vitro.

Protección del daño oxidativo del ADN, con un efecto significativo sobre los niveles de MDA, ex vivo. as 
En la tabla 3 se resume el diseño y resultados de varios estudios en que se analizó la actividad antiagregante plaquetaria del tomate.

\section{EFECTOS DEL PROCESAMIENTO Y} ALMACENAMIENTO DEL TOMATE

El actual estilo de vida ha favorecido el consumo de alimentos procesados (58). Así, las preparaciones elaboradas con tomate (salsa, pasta, sopa, jugo y conserva) son componentes importantes de la dieta $(59,60)$.

La estabilidad de los componentes del tomate y sus propiedades fisicoquímicas se deben a las condiciones de producción, como: cantidad de agua, temperatura, luz, isomerización, oxidación y $\mathrm{pH}(61,62)$. También pueden influir los materiales de embalaje y las condiciones de almacenamiento (63).

\section{a) Efecto del procesamiento Salsa de tomate}

El licopeno puede encontrarse como isómeros cis y trans (64). Los primeros son más abundantes en los productos procesados (salsa, sopa y ketchup) que en los frescos (65) y presentan mayor absorción $(64,66,67)$. En el plasma y tejidos humanos, el licopeno se encuentra preferentemente en su forma cis (68).

\section{Tomate deshidratado}

Se ha observado que el mejor tratamiento para obtener un deshidratado a partir de jugo de tomate es someterlo a $180^{\circ} \mathrm{C}$ y $100 \%$ de maltodextrina; así el licopeno presenta mayor estabilidad y el producto posee mejores propiedades de color y humedad (69). Debido a la pérdida de agua durante el procesamiento térmico, el contenido de licopeno, beta-caroteno y alfa-tocoferol aumenta en función del peso húmedo (70), confiriendo una función protectora a estos productos (71).

El contenido de licopeno aumenta a diferentes temperaturas de secado $\left(40,80\right.$ y $\left.120^{\circ} \mathrm{C}\right)$ durante los primeros 60 minutos (72). Así, el tratamiento térmico mejora el valor nutritivo de los derivados del tomate, al aumentar la absorción de cis-licopeno y con ello la actividad antioxidante $(73,74)$. Por su parte, el contenido de vitamina $\mathrm{C}$ de los derivados de tomate (tomate al horno, salsa y sopa) disminuye durante el procesamiento térmico, correspondiendo al antioxidante más lábil y más afectado por las condiciones de almacenamiento (75).

\section{Tomates en conserva}

Durante el proceso de industrialización de los tomates en conserva, la etapa de extracción de la piel por escaldado provoca una disminución del contenido de licopeno, indicando que su presencia en la piel es

\section{TABLA 2}

Estudios que muestran efectos hipolipemiantes del tomate.

\begin{tabular}{|c|c|c|c|c|}
\hline Sujetos/animales & Tomate o derivados & Determinaciones & Resultados & Referencia \\
\hline $\begin{array}{l}21 \text { sujetos sanos } \\
(\mathrm{IMC}=23,5 \pm 2,3 \\
\mathrm{kg} / \mathrm{m} 2)\end{array}$ & $\begin{array}{l}\text { Consumo de } 400 \mathrm{~mL} \text { de jugo } \\
\text { de tomate y } 30 \mathrm{mg} \text { de } \\
\text { Ketchup al día por } 3 \text { semanas. }\end{array}$ & $\begin{array}{l}\text { Actividad } \\
\text { hipolipemiante. }\end{array}$ & $\begin{array}{l}\text { Disminución de } \\
\text { colesterol-LDL. }\end{array}$ & 50 \\
\hline $\begin{array}{l}17 \text { sujetos } \\
\text { sanos. }\end{array}$ & $\begin{array}{l}\text { Consumo de jugo de tomate } \\
\text { ( } 4 \mathrm{~mL} / \mathrm{kg} \text { /día) durante } 7 \text { días. }\end{array}$ & $\begin{array}{l}\text { Lipoproteínas } \\
\text { plasmáticas. }\end{array}$ & $\begin{array}{l}\text { HDL y licopeno aumen- } \\
\text { taron } 9,47 \% \text { y } 48,27 \% \text {, } \\
\text { respectivamente, in vivo. }\end{array}$ & 51 \\
\hline $\begin{array}{l}24 \text { sujetos sanos } \\
(\mathrm{IMC}=21,5 \pm 2,8 \\
\mathrm{kg} / \mathrm{m} 2)\end{array}$ & $\begin{array}{l}\text { Consumo de } 250 \mathrm{~mL} \text { de jugo } \\
\text { de tomate o jugo de tomate } \\
\text { enriquecido con vitamina } \mathrm{C} \text {. }\end{array}$ & $\begin{array}{l}\text { Biomarcadores } \\
\text { de estrés } \\
\text { oxidativo. }\end{array}$ & $\begin{array}{l}\text { Reducción del colesterol } \\
\text { total, proteína } C \text { reactiva } \\
\text { y estrés oxidativo, in vivo. }\end{array}$ & 49 \\
\hline $\begin{array}{l}10 \text { ratas wistar } \\
\text { macho }(200-300 \mathrm{~g}) \\
\text { y } 10 \text { ratas wistar } \\
\text { hembra }(280-470 \mathrm{~g}) .\end{array}$ & $\begin{array}{l}\text { Consumo de extracto de } \\
\text { pulpa de tomate fresco } \\
\text { durante } 15 \text { días. }\end{array}$ & $\begin{array}{l}\text { Nivel de } \\
\text { colesterol total. }\end{array}$ & $\begin{array}{l}\text { Reducción del } \\
\text { colesterol total. }\end{array}$ & 47,48 \\
\hline
\end{tabular}


importante (76). Sin embargo, luego se observa un aumento significativo en concentración de licopeno hasta la obtención del producto terminado, lo que indicaría que el tratamiento térmico $\left(98^{\circ} \mathrm{C}, 58 \mathrm{~min}\right)$ actúa sobre las paredes celulares, liberando el carotenoide (77).

El tomate en conserva presenta mejor absorción de licopeno que el fresco, cerca de cuatro veces más (78), mejorando así su capacidad antioxidante (79) .

\section{b) Efecto del almacenamiento}

En términos generales la actividad antioxidante del tomate, evaluada en contenido de $\beta$-caroteno y ácido ascórbico, tiene una evolución positiva durante su crecimiento, almacenamiento, maduración y comercialización (80).

El almacenamiento del jugo de tomate en botellas translúcidas produce cambios fisicoquímicos y organolépticos por acción de la luz (81). Por otra parte, si el jugo se almacena en botellas de vidrio, independiente de la temperatura, presenta pérdidas significativas de ácido fólico (82). Sin embargo, se ha descrito que el jugo de tomate almacenado durante un año, independientemente del material de envase utilizado, puede mantener su valor nutricional en términos de composición antioxidante, dado por la estabilidad de licopeno, compuestos fenólicos y flavonoides totales (83). Sumado a esto se ha observado que el contenido de licopeno no presenta variación significativa de su concentración en conservas almacenadas a temperatura ambiente durante un año (77).

\section{PATENTES RELACIONADAS A EFECTOS SALUDABLES DEL TOMATE}

En 2008, Medina (84) patentó un producto nutracéutico (alimentos que proveen efectos benéficos para la salud)(85), que incluye por cada $20 \mathrm{~mL}$, ácido oleico (1-15 g) y licopeno (1 y $125 \mathrm{mg}$ ), que se utiliza junto con antioxidantes de origen natural, tales como $\beta$-caroteno, retinol, fitoesteroles y tirosol. Dicho nutracéutico se prepara mezclando y homogenizando los diferentes

\section{TABLA 3}

Estudios que muestran actividad antiagregante plaquetaria del tomate.

\begin{tabular}{lllll}
\hline $\begin{array}{l}\text { Sujetos/animales } \\
\text { Plasma rico en } \\
\text { plaqueta preparado } \\
\text { a partir de sangre } \\
\text { humana. }\end{array}$ & $\begin{array}{l}\text { Tomate o derivados } \\
(20-50 \mu \mathrm{L} \text { de jugo 100\%). }\end{array}$ & $\begin{array}{l}\text { Determinaciones } \\
\text { Actividad antiagregante } \\
\text { plaquetaria de frutas y } \\
\text { hortalizas. }\end{array}$ & $\begin{array}{l}\text { Resultados } \\
\text { inhibición de la } \\
\text { agregación plaquetaria. }\end{array}$ & Referencia \\
$\begin{array}{l}\text { Ratones machos } \\
\begin{array}{l}\text { Wistar ST y } \\
\text { hembras C57BL/6 } \\
\text { (10-11 semanas). }\end{array}\end{array}$ & $\begin{array}{l}\text { Extractos de diferentes } \\
\text { tipos de tomate. }\end{array}$ & $\begin{array}{l}\text { Actividad antiagregante } \\
\text { plaquetaria del tomate. }\end{array}$ & $\begin{array}{l}\text { Inhibición de la función } \\
\text { plaquetaria, tanto in vitro } \\
\text { como in vivo. }\end{array}$ \\
$\begin{array}{l}\text { Plasma rico en } \\
\text { plaqueta preparado } \\
\text { a partir de sangre } \\
\text { humana. }\end{array}$ & Extracto de tomate. & $\begin{array}{l}\text { Actividad antiagregante } \\
\text { plaquetaria de extracto } \\
\text { acuoso de tomate. }\end{array}$ & $\begin{array}{l}\text { Inhibición de la } \\
\text { agregación plaquetaria, } \\
\text { sin aumento de los niveles } \\
\text { basales de AMPc. }\end{array}$ \\
$\begin{array}{l}\text { 23 sujetos sanos, } \\
\text { con función } \\
\text { plaquetaria normal. }\end{array}$ & $\begin{array}{l}\text { Una dosis de bebida } \\
\text { (equivalente a } 6 \text { tomates). }\end{array}$ & $\begin{array}{l}\text { Actividad antiagregante } \\
\text { plaquetaria. }\end{array}$ & $\begin{array}{l}\text { Inhibición de la función } \\
\text { plaquetaria, ex vivo. }\end{array}$ \\
$\begin{array}{l}\text { 90 sujetos sanos, } \\
\text { con función } \\
\text { plaquetaria normal. }\end{array}$ & $\begin{array}{l}\text { Suplemento de extractos } \\
\text { de tomates equivalente } \\
\text { a 2 y } 6 \text { tomates. }\end{array}$ & $\begin{array}{l}\text { Actividad antiagregante } \\
\text { plaquetaria. }\end{array}$ & $\begin{array}{l}\text { Después de 3 horas, } \\
\text { inhibición de la agregación } \\
\text { plaquetaria, ex vivo. }\end{array}$ \\
\hline $\begin{array}{l}\text { AMPc, Adenosín monofosfato cíclico. } \\
56\end{array}$
\end{tabular}


compuestos y se administra por vía oral. Está especialmente indicado en la prevención de ECV y en procesos inflamatorios, fenómeno que es inhibido en forma sinérgica por los compuestos antes mencionados $(86,87)$.

Por otra parte, agentes naturales antitrombóticos son de gran importancia en la prevención primaria de ECV (55). Dutta-Roy patentó un producto que contiene compuestos activos solubles obtenidos desde extractos de tomates por cromatografía en columna (88). Los compuestos (mezcla de nucleósidos) inhiben la agregación inducida por ADP y colágeno (88).

Por su parte, Crosbie y O'Kennedy patentaron un extracto acuoso de tomate o una fracción activa del mismo, con propiedades para disminuir los niveles séricos de triglicéridos y prevenir trombosis (89). El citado extracto o fracción activa del mismo no contiene licopeno (89).

En cuanto al síndrome metabólico, que implica resistencia a la insulina, intolerancia a la glucosa, dislipidemia, hipertensión y obesidad (90), Petyaev y Bash patentaron el efecto hipolipemiante, hipoglicemiante y antihipertensivo del licopeno obtenido de tomates (91).

\section{CONCLUSIÓN}

El consumo regular de tomates presenta efectos: antioxidantes, hipolipemiantes y antiagregante plaquetario. Por otra parte, el procesamiento de tomates no deteriora mayormente su actividad biológica e incluso mejora la biodisponibilidad del licopeno. Los efectos del procesamiento sobre la actividad antioxidante del tomate han sido bien estudiados, no así los efectos sobre su actividad hipolipemiante y especialmente sobre su efecto antiplaquetario. En otro orden, se han desarrollado formulaciones que concentran los principios activos del tomate, con las propiedades biológicas mencionadas.

\section{RESUMEN}

Las enfermedades cardiovasculares (ECV) son la principal causa de muerte en el mundo. En su prevención tiene mucha importancia el no fumar, realizar actividad física y consumir alimentos saludables. En este contexto, numerosos estudios han demostrado la importancia del consumo frecuente de frutas y hortalizas (al menos 5 porciones al día). Se ha descrito una relación inversa entre su ingesta y el riesgo de desarrollar ECV, lo que se explica principalmente por su actividad antioxidante, hipolipemiante y en algunos casos antiplaquetaria. En ese sentido aumentar el consumo actual de tomate (Solanum lycopersicum L.) y productos del tomate, puede mejorar algunos parámetros cardiovasculares. El actual estilo de vida induce a las personas a consumir alimentos procesados, lo que podría afectar la estabilidad de sus componentes y sus propiedades fisicoquímicas. Esta revisión aborda la actividad antioxidante, hipolipemiante y antiagregante plaquetaria del tomate, como también el efecto que tiene el procesamiento y almacenaje sobre dichas actividades. Adicionalmente se resumen algunas patentes asociadas a efectos beneficiosos en la salud. Como fuentes hipliográficas se utilizó principalmente www.pubmed.org los términos utilizados en la búsqueda fueron: antiplatelet, tomato, platelet, antioxidant, entre otros; luego se buscaron los textos completos de los artículos que interesaban.

Palabra clave: Tomates, antioxidante, hipolipemiante, antiagregante plaquetario, enfermedades cardiovasculares, procesamiento, patentes.

Dirigir la correspondencia a:

Profesor

Iván Palomo G., PhD

Departamento de Bioquímica Clínica

e Inmunohematología.

Facultad Ciencias de la Salud,

Universidad de Talca,

Talca, Chile

Casilla: 747, Talca, Chile

Teléfono: 56-71-200493

Fax: 56-71-200488

E-mail: ipalomo@utalca.cl

\section{BIBLIOGRAFÍA}

1. Brown D. Mokdad A. Walke H. As'ad M. Al-Nsour M. Zindah M. Arqoob K. Belbeisi A. Projected Burden of Chronic. Noncommunicable Diseases in Jordan. Preventing Chronic Dis. 2009; 6:1-3.

2. WHO. World Health Organization. Informe sobre la salud en el mundo. Technical Report Series. Geneve. 2002.

3. Drouet L. Atherothrombosis as a systemic disease. Cerebrovascular Dis. 2000; 13:1-6.

4. Gimbrone M. Topper J. Nagel T. Anderson K. Garcia-Cardena G. Endothelial dysfunction, hemodynamic forces, and atherogenesis. Ann N Y Acad Sci. 2000; 902:230-9; discussion 239-40.

5. Badimon L. Vilahur G. Platelets, arterial thrombosis and cerebral ischemia. Cerebrovasc Dis. 2007; 24:30-9.

6. Llodrá J. Angeli V. Liu J. Trogan E. Fisher E. Randolph G. Emigration of monocyte-derived cells from atherosclerotic lesions characterizes regressive, but not progressive, plaques. Proc Nat Acad Sci. 2004; 101:11779-84.

7. Libby P. Ridker P. Maseri A. Inflammation and atherosclerosis. Circulation 2002; 105: 1135- 43.

8. Spagnoli L. Bonanno E. Sangiorgi G. Mauriello A. 
Role of inflammation in atherosclerosis. J Nuclear Med. 2007; 48:1800-15.

9. Palomo I. Toro C. Alarcón M. Rol of the platelets in the pathophysiology of atherosclerosis. Molecular Med Reports. 2008; 1:179-184.

10. Rodriguez C. Raposo B. Martinez-Gonzalez J. Casani L. Badimon L. Low density lipoproteins downregulate lysyl oxidase in vascular endothelial cells and the arterial wall. Arteriosclerosis, Thrombosis Vascular Biol. 2002; 22:1409-14.

11. Palomo I. Moore-Carrasco R. Alarcon M. Rojas A. Espana F. Andres V. Gonzalez-Navarro H. Pathophysiology of the proatherothrombotic state in the metabolic syndrome. Frontiers Bioscience 2010; 2:194-208.

12. Vega J. Bedregal P. Jadue L. Delgado I. Equidad de género en el acceso a la atención de salud en Chile. Rev Med Chil. 2003; 131:669-78.

13. Palomo I. Icaza G. Mujica V. Núñez L. Leiva E. Vásquez M. Alarcón M. Moyano E. Prevalencia de factores de riesgo cardiovascular clásicos en población adulta de Talca, Chile, 2005. Rev Med Chile. 2007; 135:904-12.

14. Palomo I. Gutierrez C. Alarcón M. Jaramillo J. Segovia F. Leiva E. Mujica V. Icaza G. Díaz N. Carrasco-Moore R. Increased Concentration of Plasminogen Activator Inhibitor-1 and fibrinogen in Individuals with metabolic syndrome. Molecular Med Rep. 2009; 2:253-7.

15. Mujica V. Leiva E.Icaza G. Diaz N. Arredondo M. Moore-Carrasco R.Orrego R. Vásquez M. Palomo I. Evaluation of metabolic syndrome in adults of Talca city, Chile. Nutr J. 2008; 7:1-6.

16. Palomo I. Torres G. Alarcón M. Maragaño P. Leiva E. Mujica V. Alta prevalencia de factores de riesgo cardiovascular clásicos en una población de estudiantes universitarios de la región centro-sur de Chile. Rev Esp Cardiol. 2006; 59:1099-105.

17. WHO. World Health Organization. Diet, nutrition and the prevention of chronic diseases. Report of a joint WHO/FAO expert consultation. Technical Report Series 2003; 916:34-8.

18. Dauchet L. Amouyel P. Hercberg S. Dallongeville J. Fruit and vegetable consumption and risk of coronary heart disease: a meta-analysis of cohort studies. J Nutr. 2006; 136: 2588-93.

19. Mink P. Scrafford C. Barraj L. Harnack L. Hong C. Nettleton J. Jacob D. Flavonoid intake and cardiovascular disease mortality: a prospective study in postmenopausal women. Am J Clin Nutr. 2007; 85:895-909.

20. Sesso H. Liu S. Gaziano J. Buring J. Dietary lyco- pene, tomato-based food products and cardiovascular disease in women. J Nutr 2003; 133:2336-41.

21. Torres C. Guzmán L. Moore-Carrasco R. Palomo I. Efecto antitrombótico, una característica poco conocida de las frutas y hortalizas. Rev Chil Nutr. 2008; 35:10-17.

22. Rissanen T. Voutilainen S. Nyyssonen K. Salonen R. Kaplan G. Salonen J. Serum lycopene concentrations and carotid atherosclerosis: the Kuopio ischaemic heart disease risk factor study. Am J Clin Nutr. 2003; 77:133-38.

23. Nuez, F. El cultivo del tomate. Mundi-Prensa. Barcelona, 1995.

24. INE. Instituto Nacional de Estadística. VI Censo Nacional Agropecuario. Santiago (Chile) 1997.

25. Dorais M. Ehret D. Papadopoulos A. Tomato (Solanum lycopersicum) health components: from the seed to the consumer. Phytochemistry Reviews 2008; 7:231-50.

26. Guil-Guerrero J. Rebolloso-Fuentes M. Nutrient composition and antioxidant activity of eight tomato (Lycopersicon esculentum) varieties. J Food Composition Analysis 2009; 22:123-9.

27. Porrini M. Riso P. Brusamolino A. Berti C. Guarnieri S. Visioli F. Daily intake of a formulated tomato drink affects carotenoid plasma and lymphocyte concentrations and improves cellular antioxidant protection. British J Nutr. 2005; 93:93-9.

28. Adalid A. Roselló S. Nuez F. Mejora de la calidad nutritiva en tomate: búsqueda de nuevas fuentes de variabilidad con alto contenido en carotenoides y vitamina C. Soc Española Ciencias Hortícolas 2007; 48:121-24.

29. Cámara H. Innovation in processing tomato: the lab and the field. Acta Horticulturae 2007; 758:97-102.

30. Saavedra D. Escaff G. Cortacáns P. Ruiz-Tagle C. Recent Development in Processing Tomato Production in Chile. Acta Horticulturae 2006; 724:335-8.

31. Saavedra D. González M. Seminario internacional: Producción de tomate para procesamiento. Serie Actas Instituto de Investigaciones Agropecuarias. Santiago (Chile) 2006.

32. Lugasi A. Hovari J. Bíró L. Brandt S. Helyes L. Factors influencing lycopene content of foods, and lycopene intake of Hungarian population. Magyar Onkologia. 2004; 48:131-36.

33. Gitenay D. Lyan B. Rambeau M. Mazur A. Rock E. Comparison of lycopene and tomato effects on biomarkers of oxidative stress in vitamin $\mathrm{E}$ deficient rats. European J Nutr. 2007; 46:468-75.

34. Hadley C. Clinton S. Schwartz S. The consumption of processed tomato products enhances plasma ly- 
copene concentrations in association with a reduced lipoprotein sensitivity to oxidative damage. J Nutr. 2003; 133:727-32.

35. Agarwal S. Rao A. Tomato lycopene and its role in human health and chronic diseases. Canadian Med Assoc J. 2000; 163:739-44.

36. Visioli F. Riso P. Grande S. Galli C. Porrini M. Protective activity of tomato products on in vivo markers of lipid oxidation. European J Nutr. 2003; 42:201-206.

37. Palomo I. Gutiérrez M. Astudillo L. Rivera C.Torres C. Guzmán L. Moore-Carrasco R. Carrasco G. Alarcón M.. Efecto antioxidante de frutas y hortalizas de la zona central de Chile. Rev Chil Nutr. 2009; 36:152-8.

38. Hussein L. Medina A. Barrionnevo A. LammuelaRaventos R. Andres-Lacueva C. Normal distribution of urinary polyphenol excretion among Egyptian males 7-14 years old and changes following nutritional intervention with tomato juice (Lycopersicon esculentum). Internat J Food Sci Nutr. 2009; 60:302-11.

39. George B. Kaur C. Khurdiya D. Kapoor H. Antioxidants in tomato (Lycopersium esculentum) as a function of genotype. Food Chem. 2004; 84: 45-51.

40. Riso P. Visioli F. Erba D. Testolin G. Porrini M. Lycopene and vitamin $C$ concentrations increase in plasma and lymphocytes after tomato intake. Effects on cellular antioxidant protection. European J Clin Nutr. 2004; 58:1350-58.

41. Porrini M. Riso P. Lymphocyte lycopene concentration and DNA protection from oxidative damage is increased in women after a short period of tomato consumption. J Nutr. 2000; 130:189-92.

42. Porrini M. Riso P. Oriani G. Spinach and tomato consumption increases lymphocyte DNA resistance to oxidative stress but this is not related to cell carotenoid concentrations. European J Nutr. 2002; 41:95-100.

43. Basu A. Imrhan V. Tomatoes versus lycopene in oxidative stress and carcinogenesis: conclusions from clinical trials. European J Clin Nutr. 2006; 61:295-303.

44. Agarwal S. Rao A. Tomato lycopene and low density lipoprotein oxidation: a human dietary intervention study. Lipids 1998; 33:981-84.

45. Ortiz H. Giacopini M. Alfonso P. Bosch V. Efecto antioxidante del tomate (Lycopersicum esculentum Mill) en la lipoproteína de alta densidad (HDL) y en los microsomas hepático de la rata. Archivos Venezolanos de Farmacología y Terapéutica 2003; 22:46-52.
46. Sahin N. Orhan C. Tuzcu M. Sahin K. Kucuk O. The Effects of Tomato Powder Supplementation on Performance and Lipid Peroxidation in Quail. Poultry Sci. 2008; 87:276-83.

47. Bustamante L. Efecto antitrombótico del consumo de tomates y aceite de oliva extra virgen en un modelo animal. Facultad de Ciencias De La Salud, Escuela de Tecnología Médica Universidad de Talca, Talca, 2009, p 59.

48. Rubio P. Efecto antitrombótico in vivo del tomate. Facultad de Ciencias De La Salud, Escuela de Tecnología Médica Universidad de Talca, Talca, 2009, p 56.

49. Jacob K. Periago M. Böhm V. Berruezo G. Influence of lycopene and vitamin $\mathrm{C}$ from tomato juice on biomarkers of oxidative stress and inflammation. British J Nutr. 2008; 99:137-46.

50. Silaste M. Alfthan G. Aro A. Kesäniemi A. Hörkkö S. Tomato juice decreases LDL cholesterol levels and increases LDL resistance to oxidation. British J Nutr. 2007; 98:1251-58.

51. Madrid E. Vásquez D. Leyton F. Mandiola C. Escobar J. El consumo de Lycopersicum esculentum podría aumentar lipoproteínas de alta densidad (HDL) y disminuir el estrés oxidativo a corto plazo. Rev Méd Chil. 2006; 134:855-62.

52. Yamamoto J. Taka T. Yamada K. Ijiri Y. Murakami M. Hirata Y. Naemura A. Hashimoto M. Yamashita T. Oiwa K. Seki J. Suganuma H. Inakuma T. Yoshida T. Tomatoes have natural anti-thrombotic effects. $\mathrm{Br}$ J Nutr. 2003; 90:1031-8.

53. Palomo I. Torres C. Guzmán L. et al. Actividades antitrombótica y antioxidante, in vitro, de extractos de frutas y hortalizas que se consumen en la región central de Chile. XXV Congreso de la Sociedad Española de Trombosis y Hemostasis, 2009.

54. Dutta-Roy A. Crosbie L. Gordon M. Effects of tomato extract on human platelet aggregation in vitro. Platelets 2001; 12:218-27.

55. O’Kennedy N. Crosbie L. Van Lieshout M. Broom J. Webb D. Dutta-Roy A. Effects of antiplatelet components of tomato extract on platelet function in vitro and ex vivo: a time-course cannulation study in healthy humans. Am J Clin Nutr. 2006; 84:570-9.

56. O'Kennedy N. Crosbie L. Whelan S. Luther V. Horgan G. Broom J. Webb D. Dutta-Roy A. .Effects of tomato extract on platelet function: a double-blinded crossover study in healthy humans. Am J Clin Nutr. 2006; 84:561-9.

57. Lazarus S. Garg M. Tomato extract inhibits human platelet aggregation in vitro without increasing 
basal cAMP levels. Internat J Food Sci Nutr. 2004: 55:249-56.

58. Fernández C. Pitre A. LLobregat M. Rondón Y. Evaluación del Contenido de Licopeno en Pastas de Tomate Comerciales. Información Tecnológica. 2007; 18:31-8.

59. Re R. Bramley P. Rice-Evans C. Effects of food processing on flavonoids and lycopene status in a Mediterranean tomato variety. Free Radical Res. 2002; 36:803-810.

60. Djuric Z. Powell L. Antioxidant capacity of lycopene-containing foods. Internat J Food Sci Nutr. 2001; 52:143-9.

61. Nguyen M. Schwartz S. Lycopene: chemical and biological properties. Food Technol. 1999; 53:38-45.

62. Shi J. Maguer M. Lycopene in tomatoes: chemical and physical properties affected by food processing. Crit Rev Biotechnol. 2000; 20:293-334.

63. Garcia-Alonso F. Bravo S. Casas J. Perez-Conesa D. Jacob K. Periago M. Changes in Antioxidant Compounds during the Shelf Life of Commercial Tomato Juices in Different Packaging Materials. J Agric Food Chem. 2009; 57:6815-22.

64. Boileau T. Boileau A. Erdman J. Bioavailability of all-trans and cis-isomers of lycopene. Experiment Biol Med. 2002; 227:914-9.

65. Gartner C. Stahl W. Sies H. Lycopene is more bioavailable from tomato paste than from fresh tomatoes. Am J Clin Nutr. 1997; 66:116-22.

66. Agarwal A. Shen H. Agarwal S. Rao A. Lycopene content of tomato products: its stability, bioavailability and in vivo antioxidant properties. $\mathrm{J}$ Med Food. 2001; 4:9-15.

67. Van Het Hof K.De Boer B. Tijburg L. Lucius B. Zijp I. West C. Hautvast J. Weststrate J. Carotenoid bioavailability in humans from tomatoes processed in different ways determined from the carotenoid response in the triglyceride-rich lipoprotein fraction of plasma after a single consumption and in plasma after four days of consumption. J Nutr. 2000; 130:1189-96.

68. Unlu N. Bohn T. Francis D. Nagaraja H. Clinton S. Schwartz S. Lycopene from heat-induced cisisomer-rich tomato sauce is more bioavailable than from all-trans-rich tomato sauce in human subjects. British J Nutr. 2007; 98:140-6.

69. Candelas-Cadillo M. Alanís-Guzmán M. BautistaJusto M. Del Río-Olague F. García-Díaz C. Contenido de licopeno en jugo de tomate secado por aspersion lycopene content in spray-dried tomato juice. Rev Mexicana Ingeniería Quím. 2005; 4:299-307.

70. Seybold C. Frohlich K. Bitsch R. Otto K. Bohm
V. Changes in contents of carotenoids and vitamin E during tomato processing. J Agric Food Chem. 2004; 52:7005-10.

71. Takeoka G. Dao L. Flessa S. Gillespie D. Jewell W. Huebner B. Bertow D. Ebeler S. Processing effects on lycopene content and antioxidant activity of tomatoes. J Agric Food Chem 2001; 49:3713-7.

72. Chang C. Liu Y. Study on lycopene and antioxidant contents variations in tomatoes under air-drying process. J Food Sci. 2007; 72:E532-E540.

73. Dewanto V. Wu X. Adom K. Liu R. Thermal processing enhances the nutritional value of tomatoes by increasing total antioxidant activity. J Agric Food Chem. 2002; 50:3010-14.

74. Richelle M. Bortlik K. Liardet S. Hager C. Lambelet P. Baur M. Applegate L. Offord E. A food-based formulation provides lycopene with the same bioavailability to humans as that from tomato paste. $\mathbf{J}$ Nutr. 2002; 132:404-08.

75. Gahler S. Otto K. Bohm V. Alterations of vitamin $\mathrm{C}$, total phenolics, and antioxidant capacity as affected by processing tomatoes to different products. J Agric Food Chem. 2003; 51: 7962-8.

76. Reboul E. Borel P. Mikail C Abou L. Charbonnier M. Caris-Veyrat C. Goupy P. Portugal H. Lairon D. Amiot M. Enrichment of Tomato Paste with 6\% Tomato Peel Increases Lycopene and \{beta\}-Carotene Bioavailability in Men. J Nutr. 2005; 135:790-94.

77. Ordóñez A. Balanza M. Martín F. Flores C. Estabilidad del Carotenoide Licopeno en Tomates en Conserva. Información Tecnológica 2009; 20:31-7.

78. Cohn W. Thürmann P. Tenter U. Aebischer C. Schierle J. Schalch W. Comparative multiple dose plasma kinetics of lycopene administered in tomato juice, tomato soup or lycopene tablets. European J Nutr. 2004; 43:304-12.

79. Shi J. Dai Y. Kakuda Y. Mittal G. Xue S. Effect of heating and exposure to light on the stability of lycopene in tomato puree. Food Control 2008; 19:514-20.

80. Zapata L. Gerard L. Davies C. Schvab M. Estudio de los componentes antioxidantes y actividad antioxidante en tomates. Ciencia, Docencia y Tecnología. 2007; 35:175-93.

81. Xie L. Ying Y. Use of near-infrared spectroscopy and least-squares support vector machine to determine quality change of tomato juice. J Zhejiang Univ Sci B 2009; 10:465-71.

82. Iniesta M. Perez-Conesa D. Garcia-Alonso J. Ros G. Periago M. Folate Content in Tomato (Lycopersicon esculentum). Influence of Cultivar, Ripeness, Year of Harvest, and Pasteurization and Storage Tem- 
peratures. J Agric Food Chem. 2009; 57:4739-45.

83. Weisburger J. Evaluation of the evidence on the role of tomato products in disease prevention. Experiment Biol Med. 1998; 218:140-43.

84. Medina J. Product for Use in the Prevention and Treatment of Cardiovascular Diseases, Cancer and Chronic Inflammatory Diseases. Organización Mundial de la Propiedad Intelectual. Estados Unidos, 2008.

85. Waliszewski K. Blasco G. Propiedades nutraceúticas del licopeno. Salud Pública de México 2010; 52:254-265

86. Etminan M. Takkouche B. Caamaño-Isorna F. The role of tomato products and lycopene in the prevention of prostate cancer: a meta-analysis of observational studies. Cancer Epidemiology Biomarkers \&
Prevent 2004; 13:340-5.

87. Giovannucci E. Tomatoes, tomato-based products, lycopene, and cancer: review of the epidemiologic literature. J Nat Cancer Inst. 1999; 91:317-31.

88. Dutta-Roy A. Antithrombotic agents Provexis Limited. Estados Unidos, 2005.

89. O'Kennedy N. Crosbie L. Therapeutic uses of tomato extracts. Provexis natural products limited. Estados Unidos, 2009.

90. Palomo I. Moore-Carrasco R. Alarcón M. Rojas A. Mujica V. Hasbun S. Fisiopatología del estado protrombótico en el síndrome metabólico. Acta Méd Colomb. 2009; 34:80-4.

91. Petyaev I. Bash G. Lycopene for the treatment of metabolic dysfunction Cammedica limited. Estados Unidos, 2009. 